\title{
Considerations for Use of Hematopoietic Growth Factors in Patients With Cancer Related to the COVID-19 Pandemic
}

\author{
Elizabeth A. Griffiths, MD; ; Laura M. Alwan, PharmD, BCOP2; Kimo Bachiashvili, MD³; Anna Brown, PharmD, BCOP4; \\ Rita Cool, PharmD, BCOP5; Peter Curtin, MD; Mark B. Geyer, MD7; Ivana Gojo, MD; Avyakta Kallam, MD;; \\ Wajih Z. Kidwai, MD ${ }^{10}$; Dwight D. Kloth, PharmD, BCOP11; Eric H. Kraut, MD ${ }^{12}$; Gary H. Lyman, MD, MPH²; \\ Sudipto Mukherjee, MD, PhD, MPH ${ }^{13}$; Lia E. Perez, MD ${ }^{14}$; Rachel P. Rosovsky, MD, MPH ${ }^{15}$; Vivek Roy, MD ${ }^{16}$; \\ Hope S. Rugo, MD ${ }^{17}$; Sumithira Vasu, MBBS ${ }^{12}$; Martha Wadleigh, MD ${ }^{18}$; \\ Peter Westervelt, MD, PhD ${ }^{19}$; and Pamela S. Becker, MD, PhD²
}

\section{ABSTRACT}

Hematopoietic growth factors, including erythrocyte stimulating agents (ESAs), granulocyte colony-stimulating factors, and thrombopoietin mimetics, can mitigate anemia, neutropenia, and thrombocytopenia resulting from chemotherapy for the treatment of cancer. In the context of pandemic SARS-CoV-2 infection, patients with cancer have been identified as a group at high risk of morbidity and mortality from this infection. Our subcommittee of the NCCN Hematopoietic Growth Factors Panel convened a voluntary group to review the potential value of expanded use of such growth factors in the current high-risk environment. Although recommendations are available on the NCCN website in the COVID-19 Resources Section (https://www.nccn.org/covid-19/), these suggestions are provided without substantial context or reference. Herein we review the rationale and data underlying the suggested alterations to the use of hematopoietic growth factors for patients with cancer in the COVID-19 era.

J Natl Compr Canc Netw, doi: 10.6004/jnccn.2020.7610 Published online September 1, 2020

\footnotetext{
${ }^{1}$ Roswell Park Comprehensive Cancer Center, Buffalo, New York; ${ }^{2}$ Fred Hutchinson Cancer Research Center/Seattle Cancer Care Alliance, Seattle, Washington; ${ }^{3} \mathrm{O}$ 'Neal Comprehensive Cancer Center at the University of Alabama, Birmingham, Alabama; ${ }^{4}$ University of Michigan Rogel Cancer Center, Ann Arbor, Michigan; ${ }^{5}$ The University of Texas MD Anderson Cancer Center, Houston, Texas; ${ }^{6}$ UC San Diego Moores Cancer Center, La Jolla, California; ${ }^{7}$ Memorial Sloan Kettering Cancer Center, New York, New York; ${ }^{8}$ The Sidney Kimmel Comprehensive Cancer Center at Johns Hopkins, Baltimore, Maryland; ${ }^{9}$ University of Nebraska Medical Center, Fred \& Pamela Buffett Cancer Center, Omaha, Nebraska; ${ }^{10}$ Yale Cancer Center/Smilow Cancer Hospital, New Haven, Connecticut; ${ }^{11}$ Fox Chase Cancer Center, Philadelphia, Pennsylvania; ${ }^{12}$ The Ohio State University Comprehensive Cancer Center-James Cancer Hospital and Solove Research Institute, Columbus, Ohio; ${ }^{13}$ Case Comprehensive Cancer Center/University Hospitals Seidman Cancer Center and Cleveland Clinic Taussig Cancer Institute, Cleveland, Ohio; ${ }^{14}$ Moffitt Cancer Center, Tampa, Florida; ${ }^{15}$ Massachusetts General Hospital Cancer Center, Boston, Massachusetts; ${ }^{16}$ Mayo Clinic Cancer Center; Jacksonville, Florida; ${ }^{17}$ UCSF Helen Diller Family Comprehensive Cancer Center, San Francisco, California; ${ }^{18}$ Dana Farber/Brigham and Women's Cancer Center, Boston, Massachusetts; and ${ }^{19}$ Siteman Cancer Center at Barnes-Jewish Hospital and Washington University School of Medicine, Saint Louis, Missouri.
}

In December 2019, a cluster of acute respiratory illness, now known as "SARS-CoV-2-associated pneumonia" or "clinical COVID-19," emerged in Wuhan, Hubei Province, China. ${ }^{1}$ This illness has spread rapidly worldwide and as of June 12, 2020, had been reported in more than 16.5 million individuals. ${ }^{2}$ Disease presentation has been variable, with some individuals exhibiting almost no symptoms and others dying rapidly. ${ }^{3-5}$ Early reports from China on those with a more severe disease course suggested an overrepresentation of patients with cancer, although the number of patients in these series was small. ${ }^{6,7}$ Patients with severe disease presentation are more likely to die of the disease, with some series reporting extremely high mortality rates among individuals requiring ventilator support. ${ }^{4,5,8}$ Based on a concern for excess morbidity and mortality from SARS$\mathrm{CoV}-2$ infection in patients with cancer, many changes to clinical practice across the spectrum of disease have been rapidly adopted, including delays in surgery and alterations to standard chemotherapy regimens with proven clinical benefit. ${ }^{7,9,10}$ The impact that these changes in practice will have on outcomes for patients with cancer is unknown. Strategies to limit hematologic toxicity to support use of the most accepted and effective chemotherapy regimens, and thereby limit the need for changes in clinical practice, are an attractive alternative to dose reduction or omission of chemotherapy for patients with cancer who are likely to derive substantial clinical benefit from treatment. We particularly highlight the potential benefit from expanded use of hematopoietic growth factors for patients in whom chemotherapy dose reduction may compromise outcome or in whom chemotherapy dose reduction alone may not be adequate to reduce risk.

Historically, NCCN, through an expert panel of representatives from its Member Institutions, has provided supportive care guidelines updated at least 
annually for use of hematopoietic growth factors, including granulocyte-colony stimulating factors (G-CSFs), granulocyte-macrophage colony-stimulating factors, erythropoiesis-stimulating agents (ESAs), and other measures to manage cancer and chemotherapy-associated cytopenias. ${ }^{11,12}$ These guidelines are based on the best available published clinical data and expert opinion/ clinical practice at NCCN Member Institutions. Considering concern regarding increased morbidity and mortality for patients with cancer exposed to SARSCoV-2 infection, our panel convened a voluntary subcommittee to provide guidance for more optimal use of growth factors during the pandemic. Our goal from these suggestions is to reduce the risk of hematologic complications associated with cancer chemotherapy and thereby to minimize exposure to the infusion clinic, referral to the emergency department, and hospital occupancy in times when these places may be in short supply and/or pose an exposure risk for our vulnerable population. Furthermore, we suggest these recommendations to decrease the need for transfusions during times of limited blood supply due to widespread cancellation of donor blood drives. NCCN has posted the subcommittee's short-term recommendations on the COVID-19 resources page (https://www.nccn.org/covid19/pdf/HGF_COVID-19.pdf). ${ }^{13}$ We have compiled the following in-depth description of the rationale and evidence supporting our recommendations.

\section{Avoidance and Treatment of Neutropenia}

Febrile neutropenia (FN) is among the most common complications associated with chemotherapy treatment. Historically, NCCN has recommended the routine prophylactic use of G-CSFs (including FDA-approved biosimilar products) for all patients receiving chemotherapy regimens deemed to pose a high risk for development of FN $(>20 \%) .{ }^{12}$ In the context of increased risk associated with excess exposure to the healthcare setting during the pandemic (eg, urgent care centers or emergency department), the panel has extended our recommendation for routine prophylactic use of G-CSF to all patients receiving regimens deemed to pose intermediate risk $(10 \%-20 \%$ risk) or high risk of FN. We urge clinicians to remember that prophylactic G-CSFs may also be appropriate in patients receiving regimens in the low-risk category $(<10 \%)$ whose comorbidities or age place them at intrinsically higher risk for FN due to poor bone marrow reserve.

For patients presenting with FN after chemotherapy who have not previously received prophylactic treatment with PEGylated G-CSFs, we recommend that all patients be started on G-CSFs to shorten the time to neutrophil recovery. Historically, G-CSF treatment was recommended only for patients at risk for complications.
Studies of these agents have consistently demonstrated shorter times to absolute neutrophil count recovery, and the primary goal from treatment in the setting of this pandemic would be to minimize days of hospitalization. ${ }^{12}$ One caveat to this recommendation is for patients presenting with FN in the context of respiratory infection, respiratory symptoms, or confirmed or suspected COVID19. Because G-CSFs have been suggested to increase pulmonary inflammation and enhance production of inflammatory cytokines such as interleukin- 6 associated with severe COVID-19, their use might be potentially harmful in such patients. 8,14

To limit frequent visits to outpatient centers and thus the risk of exposure to SARS-CoV-2, the self-administration of daily filgrastim or long-acting pegfilgrastim (1-3 days after chemotherapy) or use of an on-body injector pegfilgrastim is preferred.

The panel agreed on the routine use of G-CSF to accelerate absolute neutrophil count recovery after autologous hematopoietic cell transplant in order to minimize days of hospitalization. ${ }^{12,15}$ Some centers are also using G-CSF after related and unrelated allogeneic transplants; those that have not adopted this approach have deferred due to concern of increased risk of graft-vshost disease. ${ }^{16}$ Use of G-CSF after cord blood and haploidentical transplants has already been included in the guidelines. ${ }^{12}$

\section{Anemia and ESAs for Mitigation of Hospital Exposure and to Overcome Potential Regional Limitations of the Blood Supply}

Blood supply shortages have been demonstrated in areas significantly impacted by pandemic infection. ${ }^{17}$ The American Association of Blood Banks has addressed suggestions to extend the blood supply in this context. ${ }^{18}$ More restrictive transfusion thresholds for red cell transfusion (eg, a threshold of $<7 \mathrm{~g} / \mathrm{dL}$ ) have been studied and found to be safe in the context of intensive care unit and hematopoietic stem cell transplant. ${ }^{19,20}$ Many centers have adopted this lower threshold for transfusion-dependent patients with hematologic and other malignancies to avoid blood shortages in the context of COVID-19. It is important to note that tolerance for this lower threshold may be limited in patients with coexisting cardiopulmonary or other conditions and that in these patients, a higher transfusion threshold or strategies to improve erythropoiesis, such as ESAs (including FDA-approved biosimilars), may be appropriate.

ESA (erythropoietin alfa and darbepoetin alfa) use for patients with cancer was historically subject to a Risk Evaluation and Mitigation Strategy (REMS) ${ }^{21}$ The REMS program for ESAs was lifted by the FDA in 2017 as a result of recognition that restrictive ESA use (ie, hemoglobin level $<10 \mathrm{~g} / \mathrm{dL}$ and dosing per prescribing information) 
was relatively safe and widely adopted by the oncologic community. The ESA REMS program had been prompted by data suggesting an increased risk for tumor progression or recurrence and higher rates of vascular adverse events (myocardial infarction, stroke, arterial/ venous thrombosis) and death. ${ }^{21}$ The NCCN Clinical Practice Guidelines in Oncology (NCCN Guidelines) for Hematopoietic Growth Factors currently recommend against ESA use in patients with cancer not receiving treatment and those receiving only minimally myelosuppressive regimens. They further discourage the use of ESAs in patients receiving therapy with curative intent. ${ }^{12}$ Despite these recommendations, not all studies confirm reduced survival with ESA use in patients with cancer. For example, the BRAVE study in metastatic breast cancer, ${ }^{22}$ a meta-analysis of darbepoetin alfa in chemotherapy-induced anemia, ${ }^{23}$ and a meta-analysis of ESAs in cancer ${ }^{24}$ have shown no adverse impact on survival from cancer progression. In light of these data and the potential for blood shortages during the COVID-19 pandemic, the panel subcommittee suggested, in the short term, broadening the use of ESA therapy with or without parenteral iron supplementation to manage anemia in patients with malignancy requiring blood transfusion support. Response to these agents can be maximized with the addition of parenteral iron supplementation for patients with functional iron deficiency (ie, transferrin saturation $<50 \%$, ferritin $<800 \mathrm{ng} / \mathrm{mL}$, or \%sat of $<20 \%$ ), and nutritional supplementation (oral vitamin $\mathrm{B}_{12}$ and folate). ${ }^{12}$

Because patients treated with ESAs to achieve a normal-range target hemoglobin level are at increased risk for thrombosis, the target for hemoglobin when using ESAs should be calibrated to avoid transfusion (ie, $\sim 9 \mathrm{~g} / \mathrm{dL})$ rather than to normalize the hemoglobin level. ${ }^{21}$ NCCN has previously published extensive recommendations regarding individuals who refuse transfusion support, and we now suggest adoption of these guidelines, including use of ESAs, nutritional supplementation of vitamin $\mathrm{B}_{12}$, folate, and iron, and limiting blood draws (reducing frequency and volume) in times of limited blood supply. ${ }^{12}$

\section{Thrombocytopenia-Related Considerations Due to Limited Blood Product Supply}

Please note that the NCCN Guidelines do not currently include a thrombopoietic mimetic (TPOm) section. ${ }^{12}$ Many institutions have decided to limit platelet transfusions to patients with active bleeding or a numerical value $<10 \mathrm{~K} / \mathrm{mcL}$. Many practitioners on our panel use prophylactic antifibrinolytics (tranexamic acid or epsilon aminocaproic acid) for those with platelet counts $<10 \mathrm{~K} / \mathrm{mcL}$ when platelets are unavailable due to blood supply shortage, or in allo-immunized patients who do not have suitable human leukocyte antigen-matched units available..$^{25}$ We recommend holding antifibrinolytics when endogenous platelet counts are $>30 \mathrm{~K} / \mathrm{mcL}$ and in patients with embolic strokes, active thromboembolism, and urinary tract bleeding.

A recent meta-analysis of TPOm for patients with chemotherapy-induced thrombocytopenia (CIT) did not show clear evidence of benefit. ${ }^{26}$ Despite the negative conclusion from this analysis, the relative risk across each of the tested parameters (particularly incidence of severe/life-threatening bleeding, bleeding of any severity, platelet count $<75 \mathrm{~K} / \mathrm{mcL}$, and overall survival) trended in favor of benefit from use of TPOm (eg, romiplostim). Evidence for benefit from TPOm was supported by a recent phase II trial of romiplostim in patients with solid tumors with CIT receiving cyclical cancer chemotherapy in whom romiplostim was shown to avoid chemotherapy delays. ${ }^{27}$ Based on the concern for CIT and bleeding in the context of a possible platelet supply shortage and the potential risk from excess exposure to the healthcare system posed by platelet transfusion, the panel recommends, in the short term, consideration of TPOm for patients with CIT. However, the committee also believed it would be best to use lower thresholds for initiation and discontinuation of romiplostim than what were used in this study in order to avoid potential adverse effects such as thrombosis. We therefore recommend initiation of TPOm for platelet counts $<30$ to $50 \mathrm{~K} / \mathrm{mcL}$ and discontinuation of TPOm when the platelet count recovers to between 75 and $100 \mathrm{~K} / \mathrm{mcL}$. We also highlight that TPOm are known to increase the risk for thrombosis and should be used with caution.

\section{Conclusions}

Hematopoietic growth factors, including ESAs, G-CSFs, and TPOm, can potentially mitigate anemia, neutropenia, and thrombocytopenia resulting from chemotherapy for cancer. In the context of pandemic SARS-CoV-2 infection and the potentially greater risks to patients with cancer and from complications related to chemotherapy treatment and exposure to the healthcare setting, our subcommittee of the NCCN Hematopoietic Growth Factors Panel urges the oncology community to carefully consider the expanded use of hematopoietic growth factors (G-CSFs, ESAs, and TPOms) in patients receiving chemotherapy regimens with intermediate to high risk of myelosuppression or in the setting of blood or platelet supply shortages. Such an approach will help mitigate excess exposure to locations of high risk, such as the emergency department and the infusion center. Furthermore, we suggest that expanded use of these growth factors will support the continued administration of effective chemotherapy for patients with cancer despite concerns from exposure to SARS-CoV-2. 
Submitted May 14, 2020; accepted for publication June 25, 2020.

Disclosures: Dr. Griffiths has disclosed that she is a scientific advisor for AbbVie, Inc., Alexion Pharmaceuticals, Inc., Boston Biomedical, Celgene Corporation/Bristol-Myers Squibb, Partner Therapeutics, and Persimmune; receives honoraria from AbbVie, Inc., Boston Biomedical, and Novartis Pharmaceuticals Corporation; receives grant/research support from Astex Pharmaceuticals and Genentech, Inc.; and receives consulting fees from Celgene Corporation and Partner Therapeutics. Dr. Alwan has disclosed that she has received honoraria from Clinical Care Outcomes and Pharmacy Times Continuing Education. Dr. Gojo has disclosed that she has served on the advisory board for Bristol Myers Squibb. Dr. Kloth has disclosed that he is a scientific advisor for Clovis Oncology, Coherus Biosciences, and Teva Pharmaceuticals. Dr. Lyman has disclosed that he receives research funds from Amgen, consulting fees from Biotheranostics, Merck, Beyond Spring, GI Therapeutics, Invitae, Mylan, Samsung, Samsung Bioepis, and Spectrum Pharmaceuticals, Inc. Dr. Mukerjee has disclosed that he is a consultant for Novartis. Dr. Rosovsky has disclosed that she has received institutional grant/ research support from Bristol Meyer Squibb Company and Janssen
Pharmaceuticals and is scientific advisor/consultant for Bristol Meyer Squibb Company, Janssen Pharmaceuticals, Portola Pharmaceuticals, and Dova Pharmaceuticals, all outside the scope of this work. Dr. Rugo hs disclosed that she receives grant/research support from Pfizer, Merck, Novartis, Lilly, Roche, Odonate, Daiichi, Eisai, Seattle Genetics, Macrogenics, Immunomedics, Sermonix; and has received consulting fees from Puma and Samsung. Dr. Becker has disclosed that she has received grant/research support from AbbVie, Inc., Amgen Inc., Bristol-Myers Squibb Company, GlycoMimetics, Inc., Novartis Pharmaceuticals Corporation, Invivoscribe, Inc., Trovagene, Secura Bio, and Pfizer Inc.; is a scientific advisor for Caremark/Accordant Health Services; and receives consulting fees from Gerson Lehrman Group. The remaining authors have disclosed that they have no financial interests, arrangements, affiliations, or commercial interests with the manufacturers of any products discussed int his article or their competitors.

Correspondence: Elizabeth A. Griffiths, MD, Center for Genetics and Pharmacology, Roswell Park Comprehensive Cancer Center, Elm \& Carlton Streets, Room 5301B, Buffalo, NY 14263. Email: Elizabeth.griffiths@ roswellpark.org

\section{References}

1. Wang D, Hu B, Hu C, et al. Clinical characteristics of 138 hospitalized patients with 2019 novel coronavirus-infected pneumonia in Wuhan, China. JAMA 2020;323:1061-1069.

2. Johns Hopkins University of Medicine. Coronavirus Resource Center. Accessed June 12, 2020. Available at: https://coronavirus.jhu.edu/map. html

3. Guan WJ, Ni ZY, Hu Y, et al. Clinical characteristics of coronavirus disease 2019 in China. N Engl J Med 2020;382:1708-1720.

4. Goyal P, Choi JJ, Pinheiro LC, et al. Clinical characteristics of Covid-19 in New York City. N Engl J Med 2020;382:2372-2374

5. Richardson S, Hirsch JS, Narasimhan M, et al. Presenting characteristics, comorbidities, and outcomes among 5700 patients hospitalized with COVID-19 in the New York City area. JAMA 2020;323:2052-2059.

6. Liang W, Guan W, Chen R, et al. Cancer patients in SARS-CoV-2 infection: a nationwide analysis in China. Lancet Oncol 2020;21:335-337.

7. Yu J, Ouyang W, Chua MLK, et al. SARS-CoV-2 transmission in patients with cancer at a tertiary care hospital in Wuhan, China [published online March 25, 2020]. JAMA Oncol, doi.org/10.1001/jamaoncol.2020.0980

8. Zhou F, Yu T, Du R, et al. Clinical course and risk factors for mortality of adult inpatients with COVID-19 in Wuhan, China: a retrospective cohort study. Lancet 2020;395:1054-1062.

9. You B, Ravaud A, Canivet A, et al. The official French guidelines to protect patients with cancer against SARS-CoV-2 infection. Lancet Oncol 2020;21: 619-621.

10. Zhang L, Zhu F, Xie L et al. Clinical characteristics of COVID-19-infected cancer patients: a retrospective case study in three hospitals within Wuhan, China. Ann Oncol 2020;31:894-901.

11. Becker PS, Griffiths EA, Alwan LM, et al. NCCN Guidelines Insights: Hematopoietic Growth Factors, Version 1.2020. J Natl Compr Canc Netw 2020;18:12-22.

12. Becker PS, Griffiths EA, Alwan LM, et al. NCCN Clinical Practice Guidelines in Oncology: Hematopoietic Growth Factors. Version 2.2020. Accessed July 22, 2020. To view the most recent version, visit NCCN.org

13. NCCN Hematopoietic Growth Factors: Short-Term Recommendations Specific to Issues with COVID-19 (SARS-CoV-2). Accessed June 12, 2020. Available at: https://www.nccn.org/covid-19/pdf/ HGF_COVID-19.pdf

14. de Araujo OR, Salomão R, Brunialti MKC, et al. Cytokine kinetics in febrile neutropenic children: insights on the usefulness as sepsis biomarkers, influence of filgrastim, and behavior of the IL-23/IL-17 pathway. Mediators Inflamm 2017;2017:8291316.
15. Trivedi M, Martinez S, Corringham S, et al. Optimal use of G-CSF administration after hematopoietic SCT. Bone Marrow Transplant 2009;43: 895-908.

16. Ringden $\mathrm{O}$, Hassan $\mathrm{Z}$, Karlsson $\mathrm{H}$, et al. Granulocyte colony-stimulating factor induced acute and chronic graft-versus-host disease. Transplantation 2010;90:1022-1029.

17. Pagano MB, Hess JR, Tsang HC, et al. Prepare to adapt: blood supply and transfusion support during the first 2 weeks of the 2019 novel coronavirus (COVID-19) pandemic affecting Washington State. Transfusion 2020;60: 908-911.

18. American Association of Blood Banks. Extending the Blood Supply - 10 Tips for Hospitals. Accessed July 10, 2020. Available at: http://www.aabb. org/advocacy/regulatorygovernment/Documents/Tips-for-HospitalsExtending-the-Blood-Supply.pdf

19. Cable CA, Razavi SA, Roback JD, et al. RBC transfusion strategies in the ICU: a concise review. Crit Care Med 2019;47:1637-1644.

20. Tay J, Allan DS, Chatelain E, et al. Liberal versus restrictive red blood cell transfusion thresholds in hematopoietic cell transplantation: a randomized, open label, phase III, noninferiority trial. J Clin Oncol 2020;38:1463-1473.

21. Aapro M, Gascón P, Patel K, et al. Erythropoiesis-stimulating agents in the management of anemia in chronic kidney disease or cancer: a historical perspective. Front Pharmacol 2019;9:1498.

22. Aapro M, Leonard RC, Barnadas A, et al. Effect of once-weekly epoetin beta on survival in patients with metastatic breast cancer receiving anthracycline- and/or taxane-based chemotherapy: results of the Breast Cancer-Anemia and the Value of Erythropoietin (BRAVE) study. J Clin Oncol 2008;26:592-598

23. Ludwig $\mathrm{H}$, Crawford $\mathrm{J}$, Osterborg $\mathrm{A}$, et al. Pooled analysis of individua patient-level data from all randomized, double-blind, placebo-controlled trials of darbepoetin alfa in the treatment of patients with chemotherapyinduced anemia. J Clin Oncol 2009;27:2838-2847.

24. Glaspy J, Crawford J, Vansteenkiste J, et al. Erythropoiesis-stimulating agents in oncology: a study-level meta-analysis of survival and other safety outcomes. Br J Cancer 2010;102:301-315.

25. Desborough MJR, Smethurst PA, Estcourt LJ, et al. Alternatives to allogeneic platelet transfusion. Br J Haematol 2016;175:381-392.

26. Zhang $X$, Chuai $Y$, Nie $W$, et al. Thrombopoietin receptor agonists for prevention and treatment of chemotherapy-induced thrombocytopenia in patients with solid tumours. Cochrane Database Syst Rev 2017;11:CD012035.

27. Soff GA, Miao Y, Bendheim G, et al. Romiplostim treatment of chemotherapy-induced thrombocytopenia. J Clin Oncol 2019;37: 2892-2898. 